\title{
A Truncated IL-17RC Peptide Ameliorates Synovitis and Bone Destruction of Arthritic Mice
}

\author{
Yuxuan Du, Yulong Tong, Wentong Mei, Junhui Jia, Menglin Niu, Wei Cao, Weiwei Lou, \\ Shentao Li, Zhanguo Li, W. Alexander Stinson, Huihui Yuan,* and Wenming Zhao*
}

Peptide-based therapy, such as modified peptides, has attracted increased attention. IL-17 is a promising therapeutic target for autoimmune diseases, and levels of circulating bioactive IL-17 are associated with rheumatoid arthritis severity. In this study, a modified truncated IL-17RC is generated to ameliorate inflammation and bone destruction in arthritis. The truncated IL-17RC binds to both IL-17A and IL-17F with higher binding capacity compared to nonmodified IL-17RC. In addition, the truncated IL-17RC reduces the secretion of inflammatory and osteoclastogenic factors induced by IL-17A/F in vitro. Moreover, the administration of truncated IL-17RC dramatically improves symptoms of inflammation and inhibited bone destruction in collagen-induced arthritis mice. Collectively, these data demonstrate that modified truncated IL-17RC peptide may be a more effective treatment strategy in the simultaneous inhibition of both IL-17A and IL-17F signaling, whereas the existing agents neutralize IL-17A or IL-17F alone. These suggest that the truncated IL-17RC may be a potential candidate in the treatment of inflammatory associated bone diseases.

leading to severe disability and increased mortality. ${ }^{[1]}$ Numerous studies have demonstrated that interleukin (IL)-17A and, to a lesser extent, IL-17F contribute to disease progression and severity in RA. ${ }^{[2,3]}$ Studies in experimental arthritis models demonstrate a detrimental role of IL-17 in the triggering of inflammatory cytokine cascades that appear to be critical in bone destruction. ${ }^{[4,5]}$ Previous studies suggest that IL-17 stimulates the secretion of other factors such as IL-6, tumor necrosis factor (TNF)- $\alpha$, and IL- $1 \beta$ in fibroblasts, osteoblasts, and osteoclasts, further aggravating the inflammation. ${ }^{[6,7]}$ Also, these cytokines affect the release of other inflammatory and osteoclastogenic factors associated with RA. ${ }^{[8-10]}$ Indeed, IL-17 has been demonstrated to induce the secretion of matrix metalloproteinases (MMPs) to enhance matrix degradation in articular cartilage explants and inhibit the synthesis of

\section{Introduction}

Rheumatoid arthritis (RA) is an immune-mediated disease characterized by inflammation and subsequent bone damage

Y. Du, Y. Tong, W. Mei, Dr. M. Niu, Dr. W. Cao, W. Lou,

Dr. S. Li, Dr. H. Yuan, Prof. W. Zhao

Department of Immunology

School of Basic Medical Sciences

Capital Medical University

No. 10 Xitoutiao, You An Men

Beijing 100069, P. R. China

E-mail: huihui_yuan@163.com; zhao-wenming@163.com

Dr. J. Jia

Department of Blood transfusion

Aerospace Center Hospital

No.15, Yuquan Road, Haidian District, Beijing 100049, P. R. China

Dr. Z. Li

Department of Rheumatology \& Immunology

Clinical Immunology Center

Peking University People's Hospital

No. 11 Xizhimen South Street, Beijing 100044, P. R. China

W. A. Stinson

Department of Internal Medicine

Division of Rheumatology

University of Michigan

Ann Arbor, MI 48109, USA

DOI: 10.1002/adhm.201600668 proteoglycan and collagen. ${ }^{[1,12]}$ Moreover, IL-17 up-regulates receptor activator nuclear factor- $\kappa \mathrm{B}$ ligand (RANKL) expression in osteoblasts, which lead to osteoclast differentiation, resulting in bone erosion. ${ }^{[13,14]}$ Therefore, blocking of IL-17 may lead to the abolishment of downstream signalling effects and thus prevent various inflammatory disorders.

Within the IL-17 family, IL-17A is most closely related to IL-17F. ${ }^{[15]}$ IL-17A and IL-17F can form homodimer complexes (AA, FF) and a heterodimer complex (AF) that can all bind to the IL-17 heterodimer receptor complex (IL-17R), consisting of IL-17RA and IL-17RC. The IL-17R induces the activation of the nuclear factor kappa-light-chain enhancer of activated $\mathrm{B}$ cells $(\mathrm{NF}-\kappa \mathrm{B})$ and mitogen-activated protein kinase (MAPK) signaling pathways to trigger transcription factors. Upon ligand binding, IL-17RC recruits the adaptor Act1/CIKS linked to TNF receptor-associated factor 6 (TRAF6) adaptor through a homotypic SEF/IL-17R (SEFIR) domain docking interaction. IL-17RC-bound Act1 oligomerizes with IL-17RAbound Act1, resulting in an effective recruitment of intermediates for efficient IL-17-dependent signal transduction. ${ }^{[15]}$ In the context of RA, both IL-17A and IL-17F mediated signalling through the IL-17R can induce the expression of proinflammatory cytokines and osteoclastogenic factors. ${ }^{[2,3]}$ Accordingly, the IL-17 pathway has emerged as a potential therapeutic target in the treatment of RA. ${ }^{[16,17]}$ Compared to IL-17RA, IL17RC binds with similarly high affinity to both IL-17A and 
IL-17F. ${ }^{[18]}$ Targeting IL-17RC may be a more effective treatment strategy in the simultaneous inhibition of both IL-17A and IL-17F signaling, whereas the existing agents neutralize IL-17A or IL-17F alone. ${ }^{[19,20]}$ A direct approach for targeting the pathway would involve the use of antibodies or modified peptides that might bind to IL-17A/F with high binding capability, and thus prevent the ligands bind to the endogenous receptors. Compared with neutralizing antibodies, modified peptides displayed some advantages in production progress and therapeutic effects, such as low molecular weight, the ability to specifically targeting, low toxicity, and easy availability. Therefore, we engineered a truncated IL-17RC peptide in prokaryotic expression system that modified the binding domain of native IL-17RC, yet lacks the transmembrane and intracellular signal transducing domains, which could block immediate intracellular signaling events, as well as more distal events such as the release of proinflammatory cytokines and chemokines to characterize it as high binding capability, low immunogenicity, dual functions, and convenient production and purification. Our aim was to evaluate whether truncated IL-17RC could ameliorate symptoms of inflammation and bone destruction in arthritic mice through inhibiting the pathogenic roles of IL-17A/F. We tested our protein's ability to inhibit cytokine release from cells and we developed two different arthritis models to test its effects in vivo. We found that truncated IL-17RC ameliorated symptoms of inflammation and bone destruction in arthritic mice through inhibiting the pathogenic roles of IL-17A/F, suggesting its potential as a drug candidate for RA.

\section{Results}

\subsection{Construction and Characterization of Truncated IL-17RC Peptide}

A recombinant pET42a-prokaryotic expression plasmid was constructed to express truncated IL-17RC peptide (Figure 1). The component and purity of this peptide were analyzed by mass spectroscopy (MS) and high performance liquid chromatography (HPLC) (Supplementary Figure 1). After ultrfiltration, the final concentration of truncated IL-17RC was $2 \mathrm{mg} \mathrm{mL}^{-1}$ (Figure 2a) and its predicted size was $\approx 19 \mathrm{kDa}$ (Figure 2b). The binding assay showed that truncated IL-17RC bound both IL-17A and IL-17F, and the absorbance value of IL-17A was higher than IL-17F. Meanwhile, the detectable levels of IL$17 \mathrm{~A} / \mathrm{F}$ were increased in a dose-dependent manner from 0.1 to $10 \mu \mathrm{g} \mathrm{mL} \mathrm{m}^{-1}$ IL-17RC (Figure 2c,d). With truncated IL-17RC as capture protein, compared with nonmodified IL-17RC, no significant differences were observed in the detectable levels between IL-17A and IL-17F (Figure 2e). Conversely, the detectable levels of the ligands significantly decreased when using nonmodified IL-17RC (Figure 2f).

\subsection{Inhibition of Truncated IL-17RC Peptide In Vitro}

In preosteoblasts (MC3T3-E1), treatment of truncated IL-17RC peptide prominently inhibited the secretion of IL- 6 by $22.89 \%$ (IL-17A) and 20.85\% (IL-17F) (Figure 3a). In parallel with IL-6 levels, IL-17RC protein also reduced TNF- $\alpha$ by $28.21 \%$ (IL-17A)

a

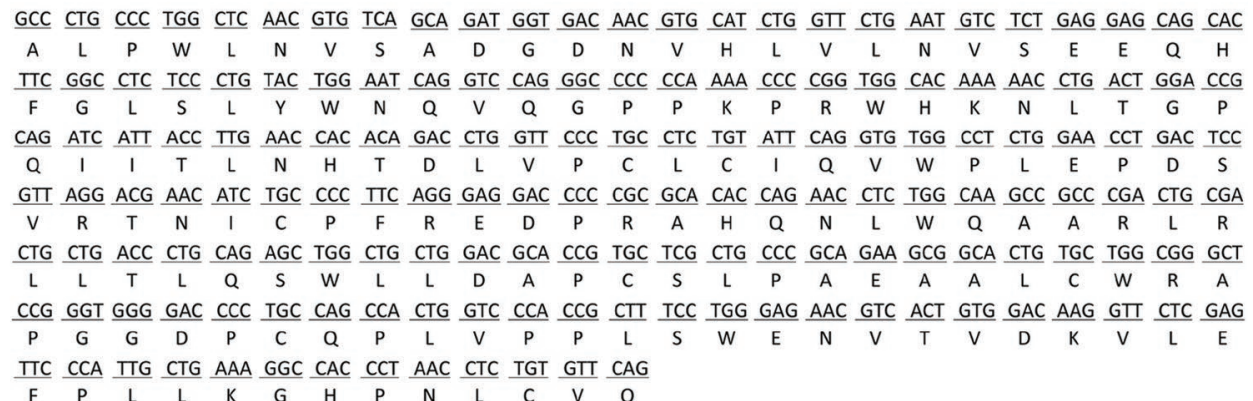

b
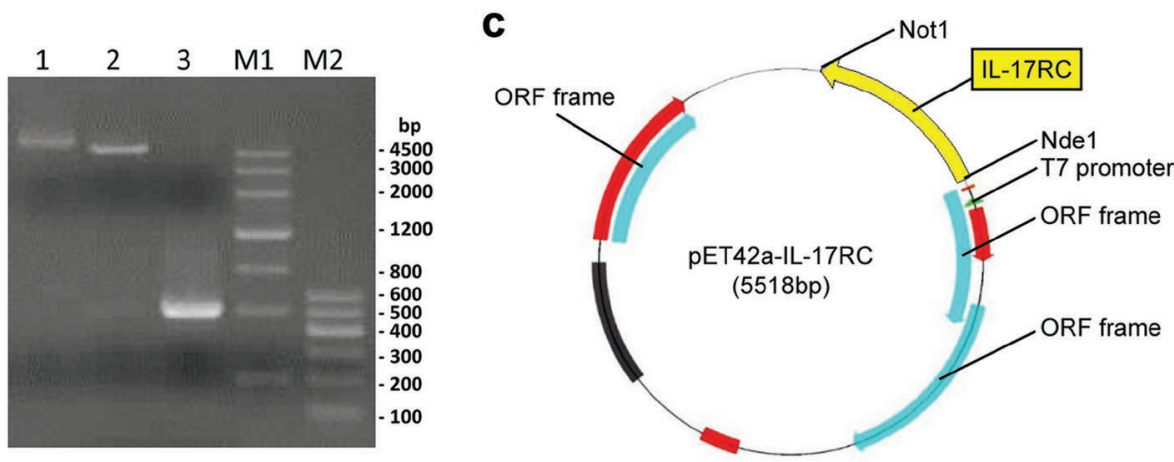

Figure 1. Construction of truncated IL-17RC peptide. a) Sequence alignment of IL-17RC exon 8 to 12. b) Agarose gel electrophoresis of undigested pET42a-IL-17RC plasmid (Lane 1), Nde I and Not I-digested recombinant plasmid (Lane 2) and pET42a-IL-17RC plasmid PCR products (Lane 3). Lane M1 and M2, DNA molecular ladder. c) pET42a-IL-17RC plasmid maps. 
a

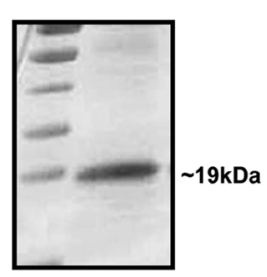

b

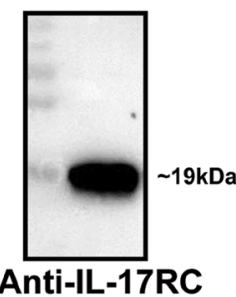

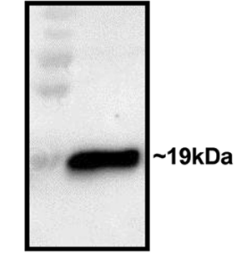

Anti-His-tag
C

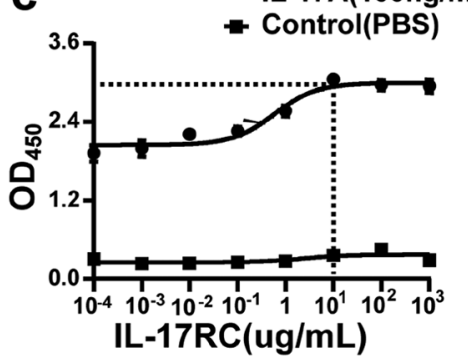

d

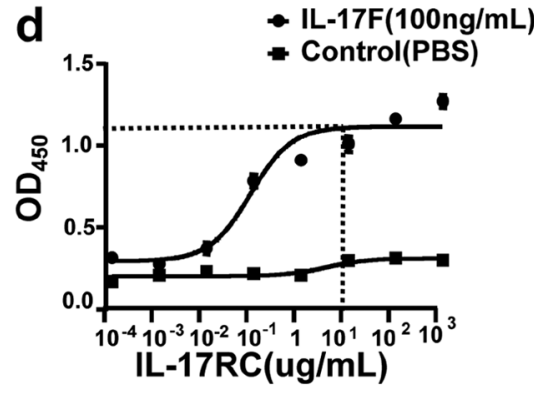

f

Non-modified IL-17RC protein
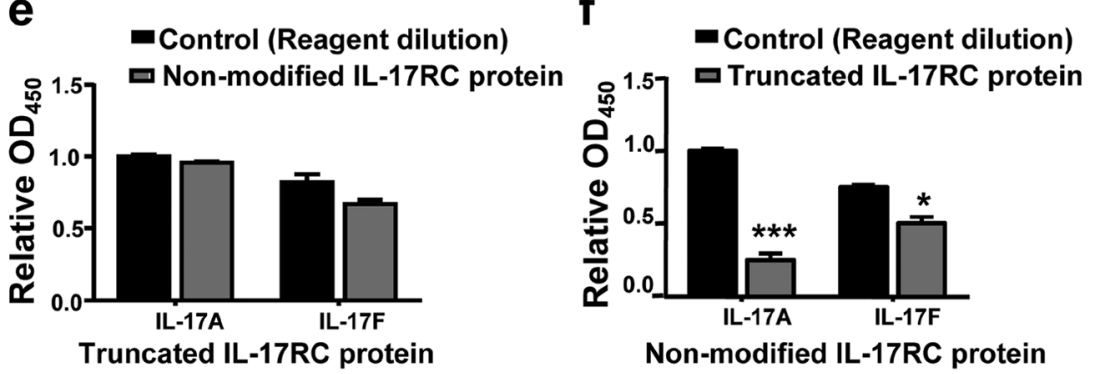

Figure 2. Preparation of truncated IL-17RC peptide. a) Expression and purity analysis of truncated IL-17RC peptide by SDS-PAGE. b) Recognition analysis of truncated IL-17RC peptide by anti-IL-17RC or anti-His-tag antibody by Western blotting. c,d) Binding assay of truncated IL17RC peptide with IL-17A or IL-17F ( $n=3$ /group). e,f) Competition assay of truncated IL-17RC peptide binding to IL-17A or IL-17F in comparison with nonmodified standard ( $n=3 /$ group). The data are expressed as the means \pm SEM, $* p<0.05$, $* * * k p 0.001$. mice than in CIA group (Figure 4b). Meanwhile, the hind-paw swelling was alleviated gradually between days 30 and 37 (Figure 4c). Compared to CIA group, IL17RC reduced synovitis, pannus formation (Figure 4d), and joint damage (Figure 4e) as determined by inflammatory (Figure $4 \mathrm{~g}$ ) and cartilage scores (Figure 4h). Severe bone erosion was observed in interphalangeal and ankle joints of CIA mice, while bone integrity was preserved in truncated IL-17RC treated group (Figure 4f). Radiographic analysis showed that BV/TV, BS/ $\mathrm{BV}, \mathrm{Ct}$. BMD, and DA were remarkably improved in the truncated IL-17RC treated group (Figure 4i-1).

\subsection{Effects of Truncated IL-17RC Peptide on Cytokine Production in CIA Mice}

Compared to the CIA group, truncated IL17RC treatment significantly reduced serum levels of IL-6, TNF- $\alpha$, monocyte chemotactic protein (MCP)-1, interferon (IFN)- $\gamma$, RANKL, and MMP-9 on day 28. Similarly, IL-6, MCP-1, RANKL, and MMP-9 expression were significantly lower on day 37 in truncated IL-17RC treated mice compared to CIA group (Figure 5a-i). In addition, osteoclastogenesis analysis with tartrate resistant acid phosphatase (TRAP) staining showed that truncated IL-17RC blocked osteoclast differentiation (Figure $5 j, k$ ) and significantly reduced the number of TRAP-positive cells (Figure 51). and $34.97 \%$ (IL-17F) (Figure 3b). Similarly, within the fibroblasts (NIH/3T3), significant reductions in the expression of IL- 6 and TNF- $\alpha$ were observed in the truncated IL-17RC group, for example, a $\approx 50 \%$ decrease in IL-6 secretion (Figure $3 \mathrm{~d}$,e).

Subsequently, IL-17RC treatment reduced the RANKL level to $52.72 \%$ in the IL-17A stimulated group or $45.63 \%$ in the IL-17F stimulated group (Figure 3c). In preosteoclasts (RAW264.7), the production of MMP-9 also showed $a \approx 30 \%$ decrease upon IL-17RC treatment (Figure 3f). After treatment with truncated IL-17RC, osteoclast differentiation was also completely prevented (Figure $3 \mathrm{~g}$ ) and the number of osteoclast-like cells was significantly reduced even in the presence of IL-17A/F in vitro (Figure $3 \mathrm{~h}$ ).

\subsection{Protection from Inflammation and Joint Damage by Trun- cated IL-17RC Peptide in Collagen-Induced Arthritis (CIA) Mice}

In the CIA model, the incidence of mice treated with truncated IL-17RC was reduced $42.86 \%$ at day 37 (Figure $4 a$ ). Moreover, the clinical scores were lower in IL-17RC-treated

\subsection{Neutralization of Truncated IL-17RC Peptide in Knee Joints}

In the CIA-IL-17A/F arthritis, truncated IL-17RC treated groups exhibited significantly decreased clinical knee scores and swelling compared to the IL-17A or IL-17F injected groups from days 29-36 (Figure 6a-d). The incidence and hind-paw clinical scores and swelling were also reduced in the truncated IL-17RC treated mice (Figure 6e), in line with previous observations (Figure $4 \mathrm{a}-\mathrm{c}$ ). Histological analysis for synovial inflammation (Figure 6f) and overall destruction of cartilage (Figure 6g) were significantly lower $\approx 40 \%$ in the truncated IL-17RC group (Figure 6j,k). Accordingly, osteoclastogenesis assays showed that truncated IL-17RC inhibited osteoclast mature (Figure 6h,i) and significantly decreased the number of osteoclast-like cells under cartilage (Figure 61).

\subsection{Prevention of Truncated IL-17RC Peptide in IL-17-Induced Bone Destruction}

In micro-CT imaging analysis, focal bone erosion, thinning of cortical bone and loss of trabecular bone were observed on the 

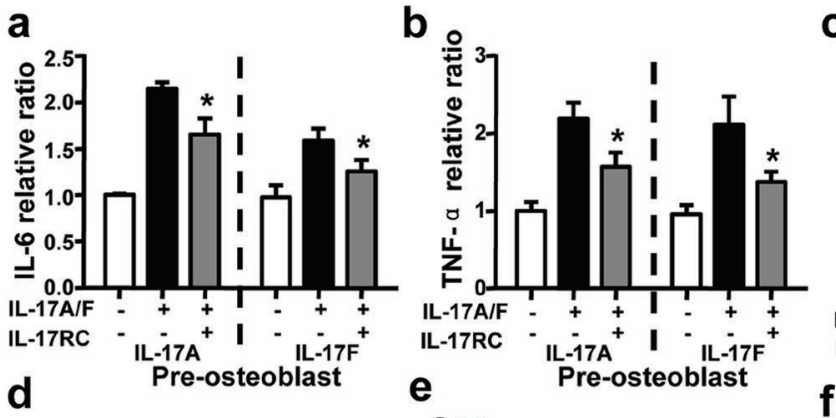

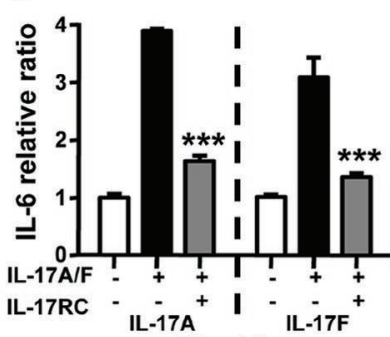

Fibroblast

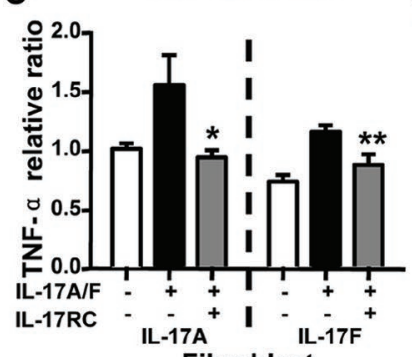

Fibroblast g

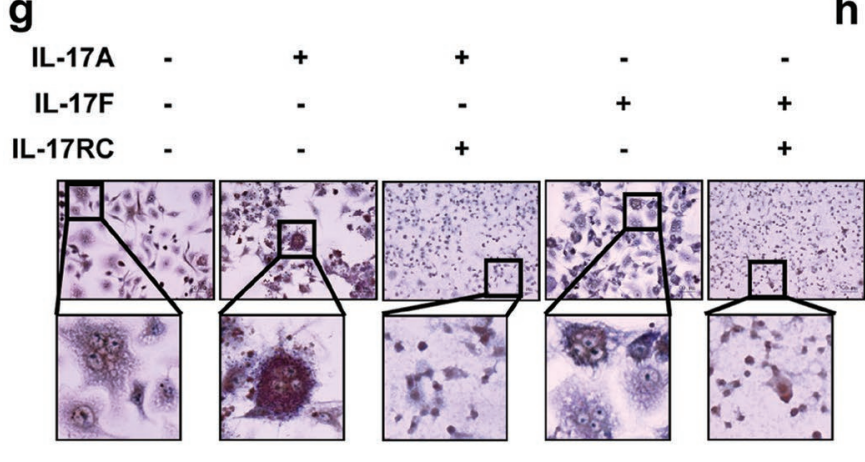

h

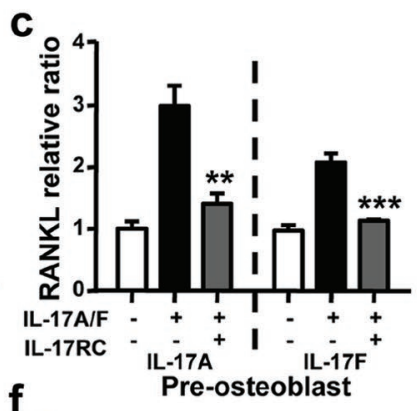

f
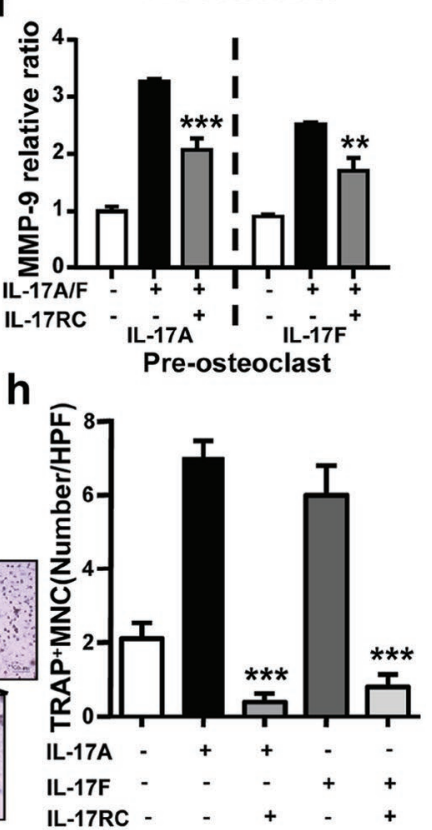

Figure 3. Inhibition of truncated IL-17RC peptide in vitro. a-f) ELISA assay of IL-6, TNF- $\alpha$, MMP-9 or RANKL in NIH/3T3, MC3T3-E1, or RAW264.7 treated with IL-17A or IL-17F with or without truncated IL-17RC peptide for $48 \mathrm{~h}$ or $7 \mathrm{~d}(n=3 /$ group). g) TRAP staining of osteoclastogenesis assay in a coculture system for RAW264.7 and MC3T3-E1 for $7 \mathrm{~d}$ with or without truncated IL-17RC peptide. Scale bar, $50 \mu \mathrm{m}$. h) The number of osteoclast-like TRAP-positive in IL-17A or IL-17F with or without truncated IL-17RC peptide ( $n=10 /$ group). The data are expressed as the means \pm SD/SEM, * $p<$ $0.05, * * 0.01, * * x p 0.001$.

2D micro-CT images in close proximity to the facet joint. In contrast, treatment with IL-17RC alleviated the above pathological changes (Figure 7a,b). Further quantitative analysis showed that Ct. BMD was also remarkably increased by 5.2\% (IL-17A) and $6.1 \%$ (IL-17F) in truncated IL-17RC treated group compared with IL-17A or IL-17F induced groups (Figure 7c). In parallel with cortical bone, a significant increase in Tb. BMD, Ct. Th, Tb. Th, and Tb. N were observed after IL-17RC treatment in IL-17A-injected mice or IL-17F-injected group (Figure 7d-g). As for $\mathrm{Tb}$. Sp, there was a $\approx 30 \%$ reduction following truncated IL-17RC treatment (Figure 7h).

\section{Discussion}

In the present study we engineered a truncated IL-17RC peptide that could bind both IL-17A and IL-17F. Our data show that truncated IL-17RC can effectively outcompete a nonmodified IL-17RC protein for the ligands IL-17A and IL-17F and reduce the secretion of inflammatory and osteoclastogenic factors induced by IL-17A/F. Moreover, the administration of truncated IL-17RC led to a dramatic improvement of inflammation and reduction of radiological bone destruction. These findings support our hypothesis that the truncated IL-17RC has a dual effect to alleviate inflammation and bone resorption through inhibiting the downstream effects of IL-17A/F.

Previous data showed that its contribution of IL-17A/F to bone erosion and synovitis and blocking of endogenous IL-17 prevented joint destruction and significantly suppressed inflammation of arthritic models. ${ }^{[21,22]}$ These findings suggest the therapeutic potential of anti-IL-17 treatment in ameliorating arthritic flares and further bone deformation. In the past ten years, a few potential therapeutic strategies targeting IL-17 have been developed, including anti-IL-17A/F antibodies, anti-IL-17R antibodies and soluble IL-17RA peptides. ${ }^{[17]}$ However, many of these studies have only looked at anti-IL-17A treatments and not IL-17A/F together. These biological products are in different stages of clinical trial for several diseases including RA, Crohn's disease, and psoriasis. ${ }^{[23]}$ Compared with neutralizing antibodies, soluble receptors can effectively compete with the 

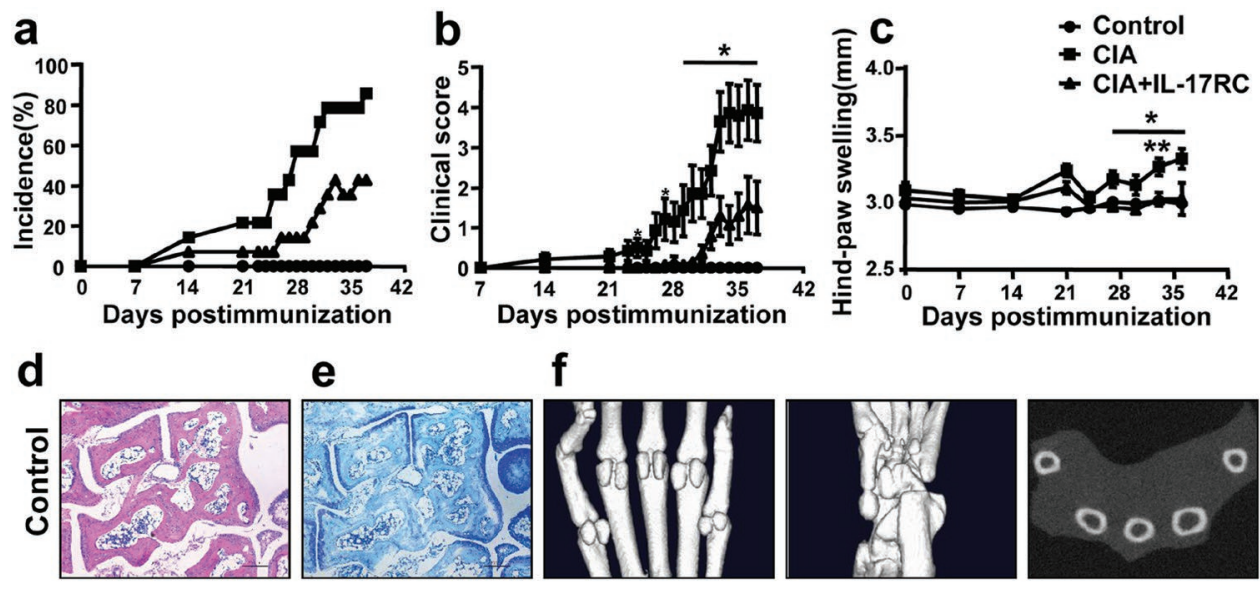

$f$
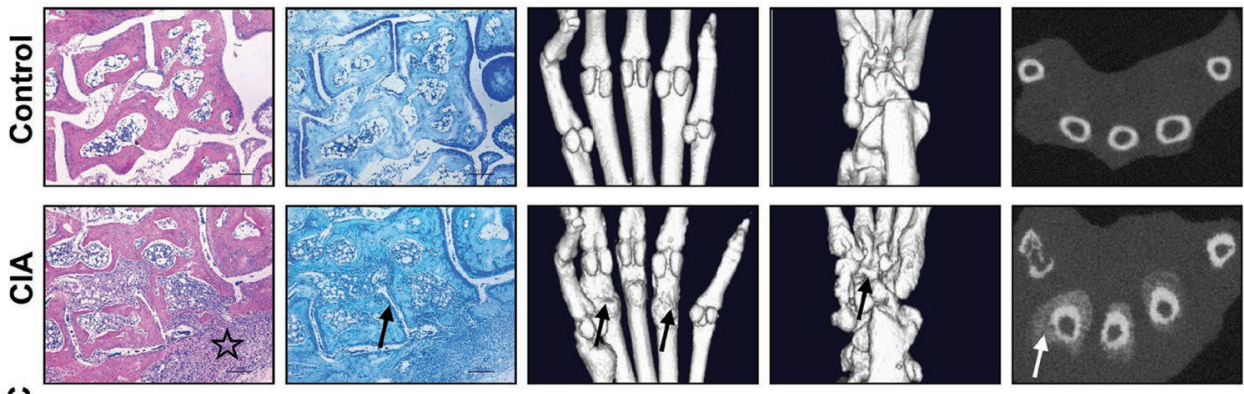

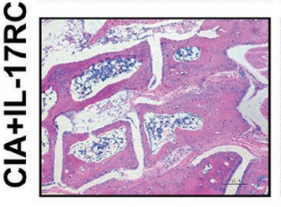

H\&E

g

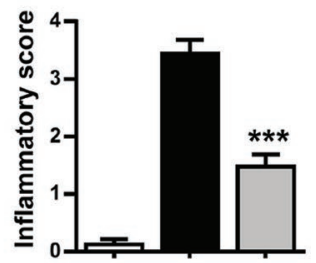

j

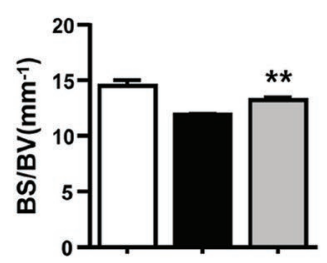

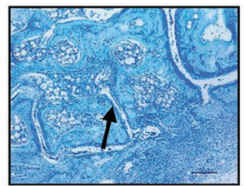
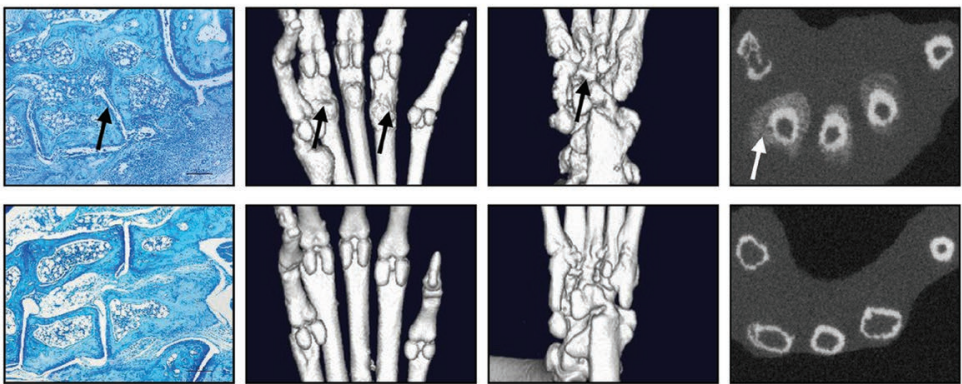

TB
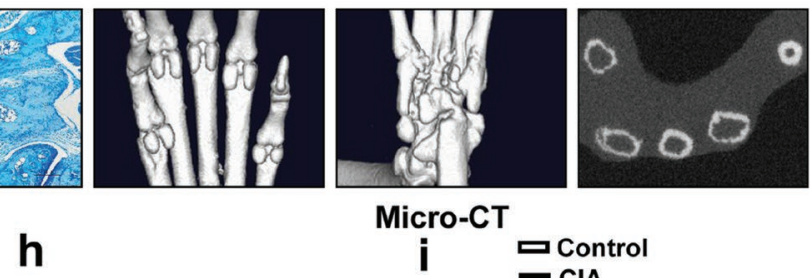

Micro-CT

i 口Control

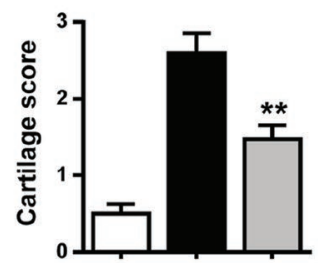

k

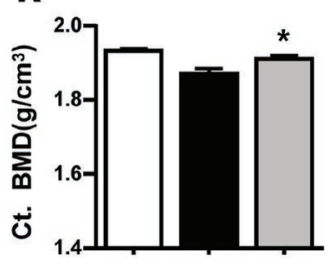

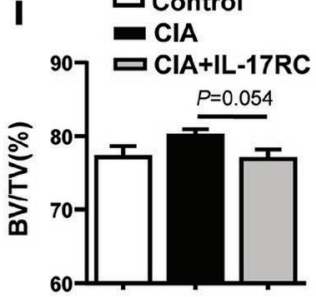

1

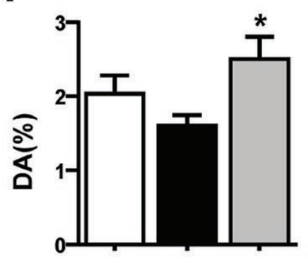

Figure 4. Protection of truncated IL-17RC peptide in CIA mice. a-c) The incidence, clinical scores and hind-paw swelling in control, CIA and truncated IL-17RC-treated C57BL/6 mice ( $n=12 /$ group). d,e,g,h) H\&E, TB staining, and histological scores of hind paw in each group ( $n=16 / g r o u p)$. Scale bar, $50 \mu \mathrm{m}$. f) Radiographic analysis of interphalangeal and carpal joints on three-dimensional micro-CT images. i-I) Bone parameters analysis of BV/TV, $\mathrm{BS} / \mathrm{BV}, \mathrm{Ct} . \mathrm{BMD}$, and DA in each group ( $n=10$ /group). The asterisk represents inflammatory cells $(\mathrm{d})$ and the arrows represent the thinning cartilage (e) and bone destruction (f).The data are expressed as the means $\pm \mathrm{SEM}, * p<0.05, * * p<0.01, * * * p<0.001$.

endogenous receptors for the native ligand binding sites. ${ }^{[24]}$ In addition, low immunogenicity makes them more suitable for application, such as Etanercept, a soluble TNF- $\alpha$ receptor. ${ }^{[25]}$ As shown from previous studies of IL-17RC splice variants, exon 8 and exon 12 are required for IL-17A/F binding to IL17RC, while exon 7 is not. ${ }^{[18]}$ Therefore, we removed the low homologous segments and unnecessary binding sequences as well as the transmembrane and intracellular signal transducing regions of IL-17RC. There was no observed loss in binding ability to IL-17A/F. At the same concentration of protein, our truncated IL-17RC peptide outcompeted a nonmodified IL17RC for the ligands IL-17A/F. This suggests that our protein could have therapeutic potential in the inhibition of IL-17A/F.

The high expression of IL-17 in the joints and synovial fluid of RA patients triggers the secretion of various proinflammatory cytokines and chemokines from synovial fibroblasts, $\mathrm{T}$ cells and macrophages, such as IL-6, IL-8, and MMPs. ${ }^{[26,27]}$ Furthermore, IL-17 also stimulates the expression of matrix MMPs and RANKL to induce cartilage destruction. We tested our protein's ability to inhibit IL-17A/F induced cytokine release 

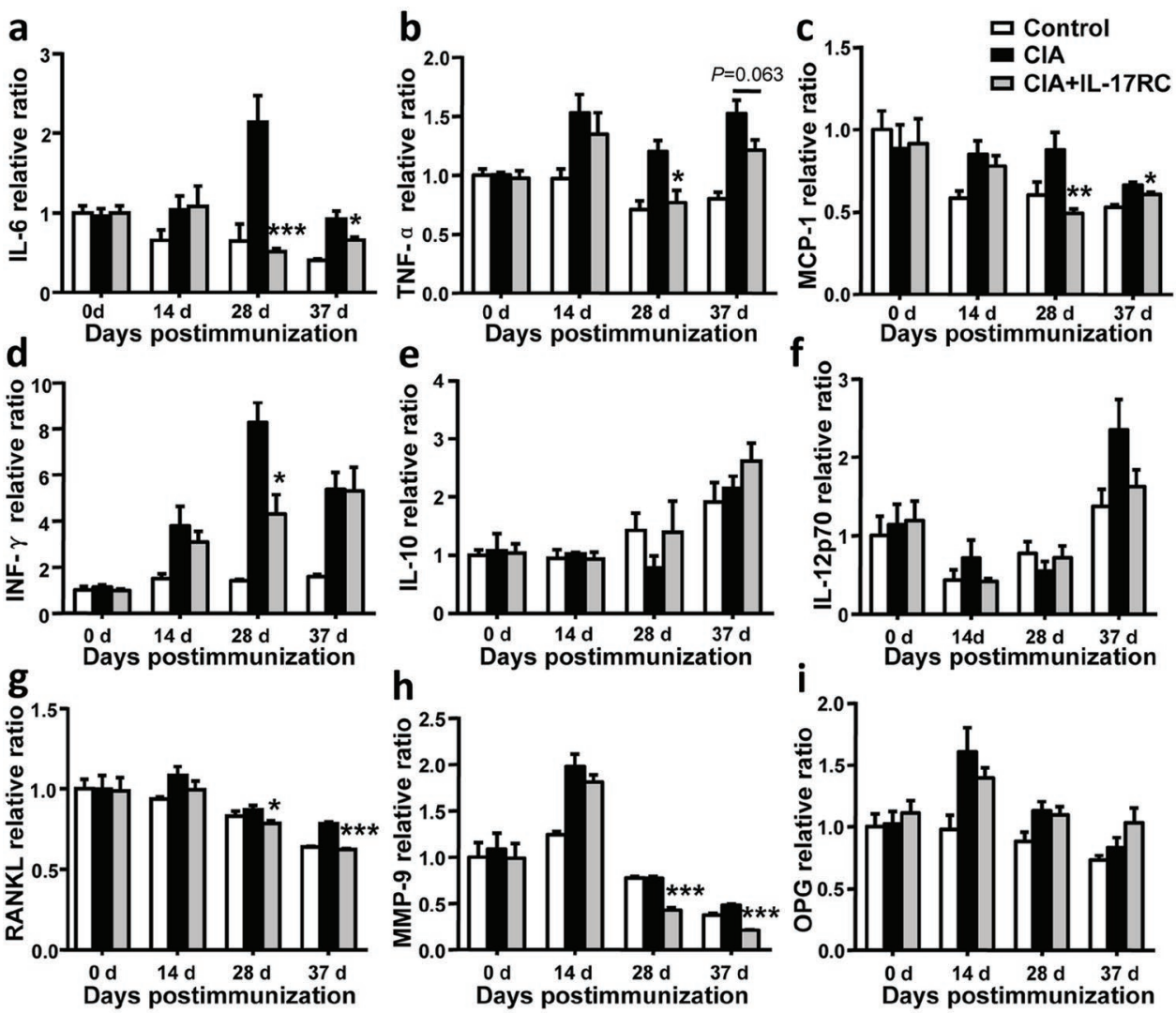

h
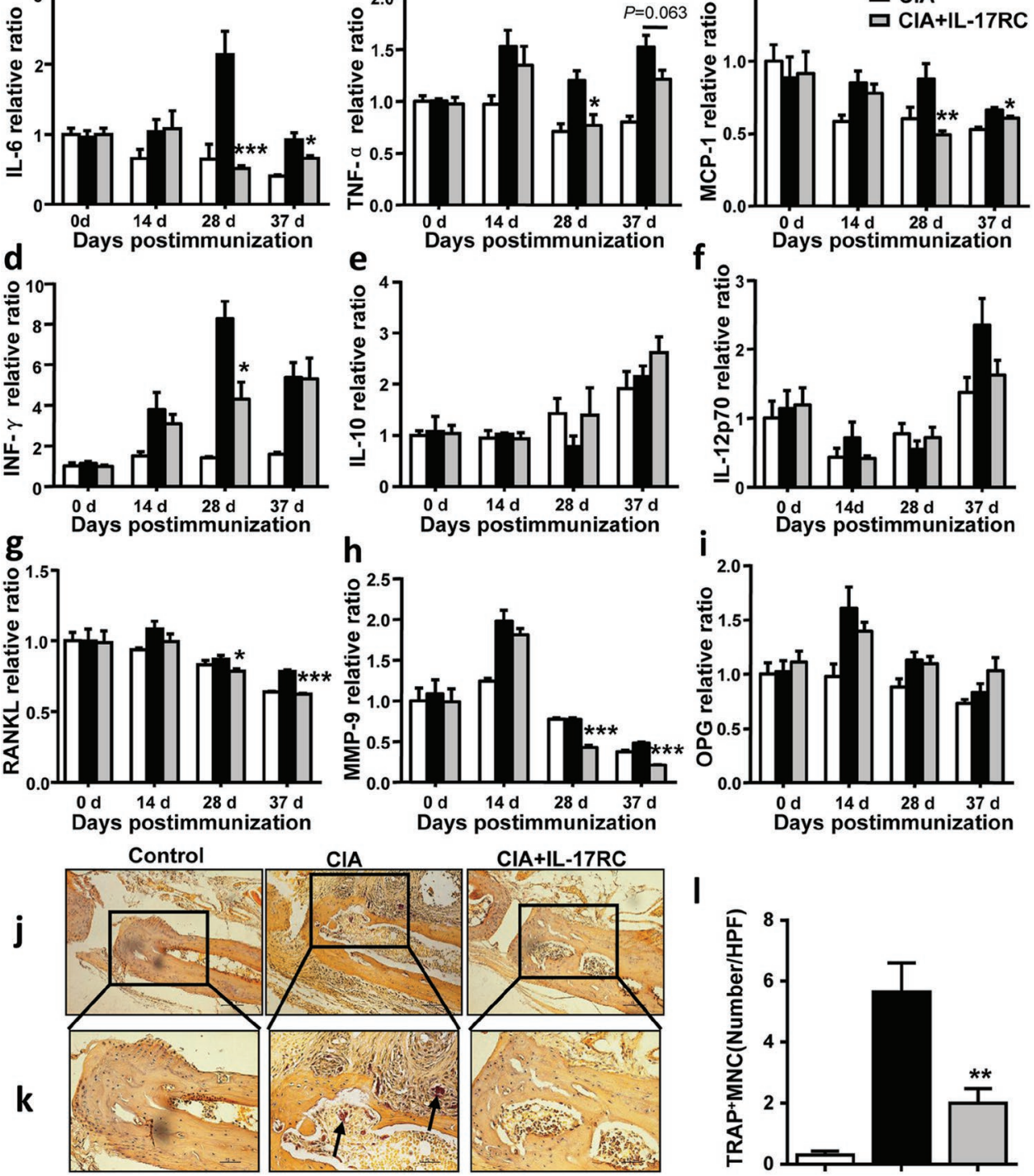

Figure 5. Interventions of truncated IL-17RC peptide in CIA mice. a-f) Cytometric bead array of IL-6, TNF- $\alpha$, MCP-1, IFN- $\gamma$, IL-10, and IL-12p70 in serum of control, CIA and truncated IL-17RC mice after immunization with chicken type II collagen for days $0,14,28$, and 37 ( $n=5 /$ group). g-i) ELISA assay of RANKL, MMP-9, and OPG in serum of each group ( $n=6 /$ group). j-k) TRAP staining of sections of tibiae derived from sacrificed animals. j) Scale bar, $100 \mu \mathrm{m}$ and k) $50 \mu \mathrm{m}$. (I) The number of osteoclast-like TRAP-positive in each group ( $n=16 /$ group). The arrows represent the mature osteoclasts (k). The data are expressed as the means \pm SEM, * $* 0.05$, ** $* 0.01$, *** $* 0.001$

from fibroblasts, osteoblasts, and osteoclasts. We found a significant decrease in the release of IL-6, TNF- $\alpha$, RANKL, and MMP-9 from these cells (Figure 3). Fortunately, similar inhibitory effects were observed despite additional injections of either IL-17A or IL-17F, indicating our protein could effectively inhibit cell surface IL-17R binding and subsequent cytokine release stimulated by either protein (Figure 5). In addition, in the preosteoclast and preosteoblast coculture system, truncated IL-17RC impaired the differentiation and maturation of osteoclasts as indicated by TRAP staining, an uncontrolled process in inflammatory diseases that results in bone deformation.

We chose the CIA model to evaluate the effect of truncated IL-17RC peptide in vivo as it shares similar immunological and pathological features with RA. ${ }^{[28]}$ In parallel with RA patients, the expression of IL-17A and IL-17F is significantly increased in the sera and in local joints of CIA mice. ${ }^{[29,30]}$ In our model, truncated IL-17RC-treated mice had greatly diminished in clinical signs and levels of the pro-inflammatory cytokines IL-6, TNF- $\alpha$, MCP-1, IFN- $\gamma$, RANKL, and MMP- 9 compared to the CIA mice (Figures 4 and 5). These results indicate that truncated IL-17RC inhibited the progression of arthritis and affirmed our in vitro findings. Furthermore, we then wanted to affirm its inhibitory effects on IL-17A and IL-17F in vivo and determine its protection of bone damage in the context of IL-17. We repeated the CIA model again, but gave additional injections of IL-17A and IL-17F into the knee joint cavity on days 28 and 32 of the model. We found that IL-17A and IL-17F significantly aggravated synovitis severity and joint damage in the arthritic mice. Treatment 


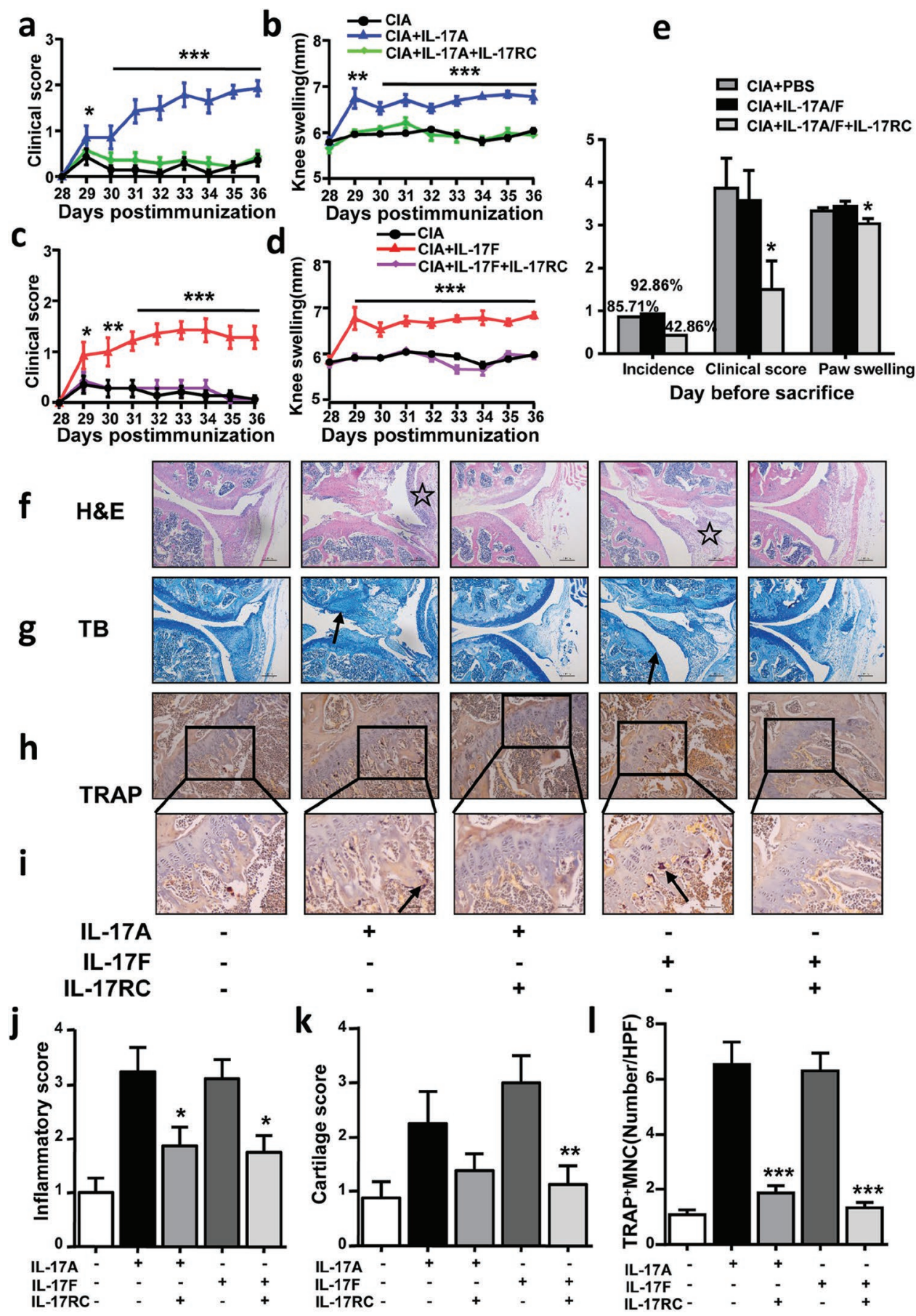

Figure 6. Neutralization of truncated IL-17RC in local joint. a-d) Clinical scores and knee swelling in CIA, IL-17A/F-injected and truncated IL-17RCtreated mice ( $n=14$ /group). e) The incidence, hind-paw clinical scores and swelling on the day before sacrifice $(n=14 / \mathrm{group})$. $\mathrm{f}-\mathrm{i}) \mathrm{H} \& \mathrm{E}$, TB, and TRAP staining of knee joints in each group. $\mathrm{f}-\mathrm{h}$ ) Scale bar, $100 \mu \mathrm{m}$ and i) $50 \mu \mathrm{m}$. j-k) Histological scores of knee joints in each group ( $n=8 /$ group). l) The number of osteoclast-like TRAP-positive in each group ( $n=8 /$ group). The asterisks represent inflammatory cells $(\mathrm{f})$ and the arrows represent the thinning cartilage (g) and mature osteoclasts (i). The data are expressed as the means \pm SEM, $* p<0.05, * * p<0.01, * * * p<0.001$.

with truncated IL-17RC, however, inhibited inflammation and prevented further bone deformation, as determined by clinical scores and radiological examination, respectively.

In fact, IL-17A and IL-17F have many overlapping functions including immunity to extracellular pathogens and neutrophil recruitment, while their cytokine knockout models indicate that
IL-17A plays a more significant role in IL-17-mediated inflammation. ${ }^{[15]}$ That might partly explain that the secretions of inflammatory cytokines were higher in IL-17A group even in the presence of truncated IL-17RC compared to IL-17F. While, the inhibition of truncated IL-17RC treatment in IL-17A group was higher compared to IL-17F group. However, no significant 

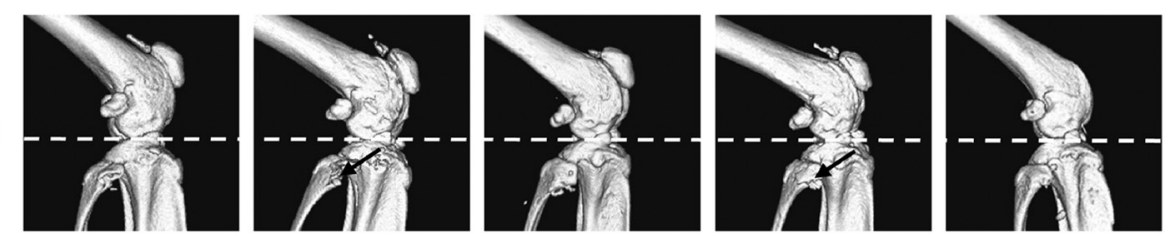

b
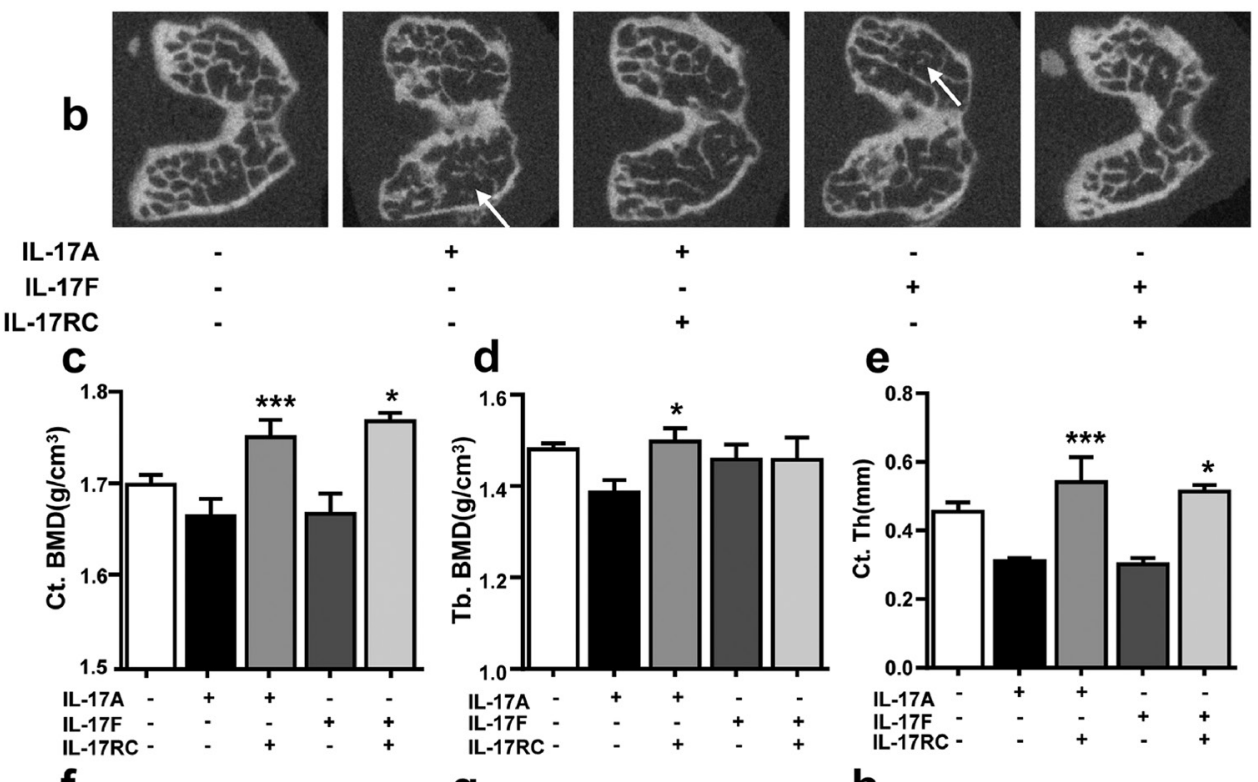

$+$

$+$

d
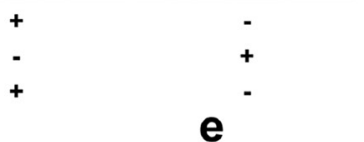

$+$

$+$
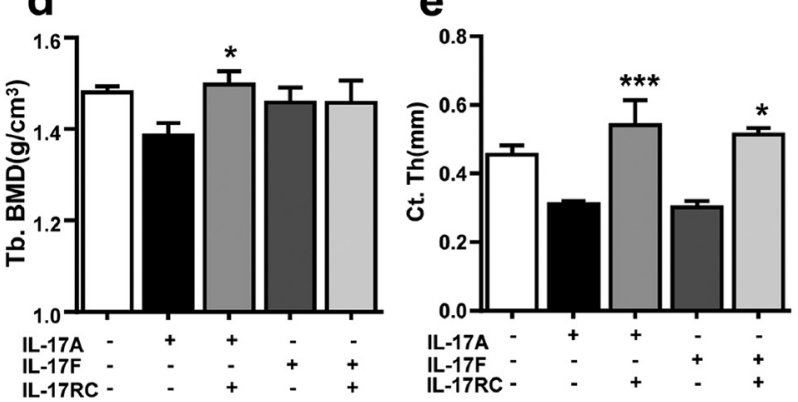

f

g
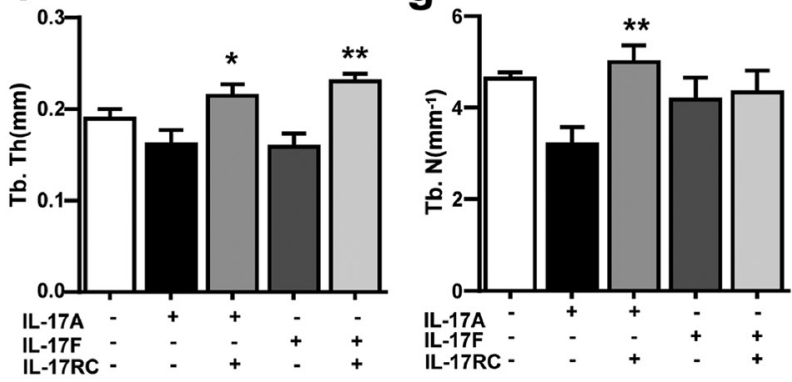

$\mathbf{h}$

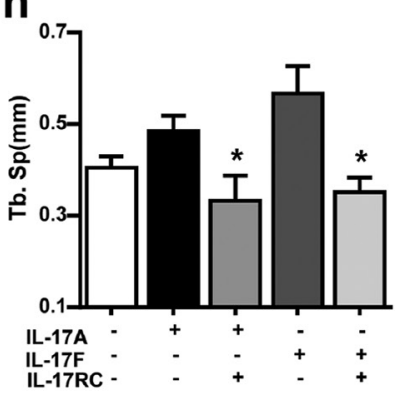

Figure 7. Prevention of truncated IL-17RC in IL-17-induced bone destruction. a,b) Radiographic analysis of knee joints on 3D micro-CT images. $c-h$ ) Bone parameters analysis of Ct.BMD, Tb.BMD, Ct.Th, Tb.Th, Tb. N, and Tb.Sp in each group ( $n=5 /$ group). The arrows represent bone destruction $(a, b)$. The data are expressed as the means \pm SEM, * $p<0.05, * * p<0.01, * * * p<0.001$.

difference was observed between IL-17A and IL-17F group after treated with truncated IL-17RC. The possible explanations were that the conditions of experiments are comparable complex to lead variable data in vitro and in vivo, such as different systems in enzyme-linked immunosorbent assay (ELISA). Besides, IL-17A and IL-17F shared some similarities in structures and pathological functions between human and mouse, because the homology of the truncated peptide (IL-17RC) is comparative of high (Supplementary Figure 2, Supporting Information).

Our results demonstrate our protein's highly effective capacity at inhibiting IL-17A/F. Not only does it bind with high affinity to its ligands, it prevents IL-17 associated cytokine release, and osteoclast maturation, findings that were corroborated in vivo. Unfortunately, we were unable to include other marketed IL-17 inhibitory drugs (such as secukinumab) as comparison or in conjunction with our protein, but our results do indicate the therapeutic potential of truncated IL-17RC. The immediate challenges that still remain includes optimizing its pharmacokinetics and prolonging the half-life (Supplementary Figure 3, Supporting Information) of truncated IL-17RC by PEGylation ${ }^{[31]}$ or other biomaterial assisted drug delivery approaches in the future.

\section{Conclusions}

In conclusion, the truncated IL-17RC peptide alleviated inflammation and bone resorption through simultaneous neutralization of IL-17A and IL-17F-driven pathogenesis in vitro and in vivo. This study highlights its high binding capability, low immunogenicity, dual functions, and convenient production and purification as a desired approach. Therefore, we anticipate this 
modified soluble receptor might serve as a potential candidate in treating IL-17 associated inflammatory diseases such as RA.

\section{Experimental Section}

Cell Lines and Mice: The murine calvarial cell line (MC3T3-E1), murine fibroblast cell line (NIH/3T3), and murine monocyte/macrophage cell line (RAW264.7) were all obtained from the Chinese Academy of Medical Sciences and Peking Union Medical College. The cell culture media were all purchased from GE Healthcare Life Sciences (South Logan, Utah, USA). The cells were separately cultured in Dulbecco's modification of Eagle's medium (DMEM)-high glucose (MC3T3-E1), DMEM-low glucose (RAW264.7), and $\alpha$-minimum essential medium (MEM) $\left(\mathrm{NIH} / 3 \mathrm{~T} 3\right.$ ) supplemented with $10 \%$ fetal bovine serum (FBS) at $37^{\circ} \mathrm{C}$ in a humidified atmosphere of $5 \% \mathrm{CO} 2$.

Male C57BL/6 mice (10 weeks old) were purchased from Beijing Vital River Laboratories (VRL) Co., Ltd. All mice were housed in a controlled environment and allowed food and water ad libitum. All experiments were conducted in accordance with the requirements of the Animal Care and Use Committee of Capital Medical University (Permit ID: SCXK-2012-0001).

Reagents: Antibodies used were as follows: goat anti-human IL-17RC antibodies and goat anti-human IL-17A or IL-17F biotinylated antibody (R\&D Systems, Minneapolis, MN, USA), mouse anti-His-tag antibody (Merck, Millipore, Darmstadt, Germany) and horseradish peroxidase (HRP)-conjugated rabbit anti-goat or mouse IgG secondary antibodies (Protein Tech Group, Inc, Chicago, USA). The recombinant proteins that were used included human IL-17A/F and IL-17RC (R\&D Systems, Minneapolis, MN, USA) and chick collagen type II (CII) (Chondrex, Morwell Diagnostic, Zurich, Switzerland). The commercial kits that were used included TRAP kit (Sigma-Aldrich, Saint Louis, MO, USA); Trizol RNA purification kit and a first strand cDNA synthesis kit (Life Technologies, California, USA); cytometric bead array (CBA) mouse inflammation kits (BD Bioscience, Allentown, PA, USA); mouse DuoSet ELISA development system and IL-6/TNF- $\alpha$ /RANKL/MMP-9/ osteoprotegerin (OPG) ELISA kits (all from R\&D Systems, Minneapolis, $M N$, USA). Freund's complete and incomplete adjuvants were obtained from Chondrex (Morwell Diagnostic, Zurich, Switzerland).

Construction of Truncated IL-17RC Peptide Expression Vector: To maximize the bioactivity of human truncated IL-17RC, a recombinant pET42a-prokaryotic expression plasmid was constructed containing the binding domain of IL-17RC spanning exons 8 to $12 .{ }^{[18]}$ The truncated IL-17RC sequence was amplified by polymerase chain reaction (PCR) from the plasmid including human IL-17RC ORF sequence (Invitrogen Life Technologies, California, USA) with the $\mathrm{N}$-terminal primer $5^{\prime}$-CGCCATATCGCCCTGCCCTGGCTCAACGT-3' (Nde I site is underlined) and the C-terminal primer 5'-ATTTGCGGCCGCCTGAACACAGAGGTTAGGGT-3' (Not I site is underlined). The PCR product was digested with NdeI and Notl, ligated into the corresponding sites of the pET-42a plasmid, and then transformed into E. coli BL21 (DE3) for protein expression. The plasmid also contains a His-tag sequence to enable purification.

Expression and Purification of Truncated IL-17RC Peptide: A 5 L Erlenmeyer flask containing $1 \mathrm{~L}$ of $2 \times Y T$ medium supplemented with $25 \mu \mathrm{g} \mathrm{mL} \mathrm{m}^{-1}$ kanamycin was inoculated with $1 \mathrm{~mL}$ of an overnight culture of E. coli BL21 (DE3) pET42a carrying the truncated IL-17RC CDNA. The flask was shaken at $250 \mathrm{rpm}$ at $37^{\circ} \mathrm{C}$ until the optical density (OD600) reached 0.6-0.8. Subsequently, isopropyl- $\beta$-D-thiogalactoside (IPTG, $0.4 \times 10^{-3} \mathrm{M}$ ) was added to the culture and the temperature was shifted to $16^{\circ} \mathrm{C}$ for $5 \mathrm{~h}$ to induce truncated IL-17RC peptide expression. Next, the cell pellets were collected by centrifugation, resuspended in precooled phosphate buffer saline (PBS) and lysed by ultrasonication in an ice-cold water bath using an ultrasonic processor (VCX-600, Sonic \& Materials, USA). The cell lysate was centrifuged at $13000 \mathrm{rpm}$ for $30 \mathrm{~min}$ at $4{ }^{\circ} \mathrm{C}$ to separate supernatant and precipitated. After filtering with $0.45 \mu \mathrm{m}$ durapore membrane filters (SLHU033RB, Merck Millipore, Germany), the supernatant was loaded onto a HisTrap affinity columns (GE Healthcare Life Technology, USA) followed by elution with an increasing gradient of $20 \times 10^{-3}, 40 \times 10^{-3}, 60 \times 10^{-3}, 80 \times 10^{-3}, 100 \times 10^{-3}$, and $500 \times 10^{-3} \mathrm{M}$ imidazole. The eluted protein was then concentrated, salts removed, and buffers exchanged using Macrosep Advance Centrifugal Device MWCO 3K (Pall Life Sciences, USA).

Identification of Truncated IL-17RC Peptide: The total protein expression was confirmed by sodium dodecyl sulfate-polyacrylamide gel electrophoresis (SDS-PAGE) and Western blotting. For SDSPAGE analysis, cell lysates were separated by $15 \%$ resolving gel and visualized by Coomassie brilliant blue R-250. For Western blotting, the sample was further transferred onto polyvinylidene fluoride (PVDF) membranes (Millipore Corporation, Billerica, MA, USA). The membrane was blocked with $5 \%$ skim milk in TBST (TBS with 0.05\% Tween-20) $2 \mathrm{~h}$ at room temperature and then incubated with a polyclonal goat antiIL-17RC antibodies $\left(0.1 \mu \mathrm{g} \mathrm{mL} \mathrm{m}^{-1}\right)$ or a monoclonal mouse anti-His-tag antibody $\left(0.1 \mu \mathrm{g} \mathrm{mL}^{-1}\right)$ overnight at $4{ }^{\circ} \mathrm{C}$. After washing three times with TBST, the membrane was incubated with HRP-conjugated rabbit antigoat IgG (1:10000) or rabbit anti-mouse IgC (1:10000) for $1 \mathrm{~h}$ at room temperature. After washing three times, the membrane was exposed to a SuperSignal West Pico ECL system (Thermo Fisher Scientific Inc., Waltham, USA) and visualized using Fusion Solo S (Vilber Lourmat, Belgium).

Binding and Competition Assays of Truncated IL-17RC Peptide: The ELISA system was developed to test the dose dependence of IL-17A or IL-17F binding to the truncated IL-17RC peptide. Ninety-six-well Maxisorp microtitre plates were coated with a 10 -fold serial dilution of truncated IL-17RC peptide $\left(10^{-3}-10^{3} \mu \mathrm{g} \mathrm{mL}-1,100 \mu \mathrm{L}\right.$ per well) overnight at $4{ }^{\circ} \mathrm{C}$. The plates were blocked with $1 \%$ bovine serum albumin (BSA) in PBS, followed by the addition of $100 \mu \mathrm{L}$ of IL-17A or IL-17F $\left(100 \mathrm{ng} \mathrm{mL}^{-1}\right)$, and incubated overnight at $4{ }^{\circ} \mathrm{C}$. After washing with PBST (PBS with $0.05 \%$ Tween-20), goat anti-human IL-17A biotinylated antibody $\left(0.2 \mu \mathrm{g} \mathrm{mL} \mathrm{L}^{-1}\right)$

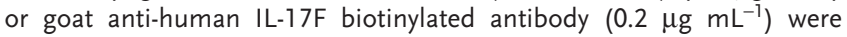
added, and the plates were incubated for $2 \mathrm{~h}$ at $37^{\circ} \mathrm{C}$. After washing, streptavidin-HRP was added to each well and the plates were incubated for $20 \mathrm{~min}$ at room temperature. After adding the substrate solution and stop solution, the absorbance was read at $450 \mathrm{~nm}$ by a microplate reader (Thermomax Technologies).

Furthermore, a modified competitive ELISA system was established to determine whether the truncated IL-17RC peptide could outcompete a nonmodified IL-17RC protein for IL-17A or IL-17F at the optimal concentration determined above. Ninety-six-well plates were coated with truncated IL-17RC peptide $\left(10 \mu \mathrm{g} \mathrm{mL} \mathrm{L}^{-1}\right)$ overnight at $4{ }^{\circ} \mathrm{C}$. The plate was blocked with $1 \%$ BSA in PBS, and IL-17A or IL-17F (100 ng mL $\left.{ }^{-1}\right)$ with or without nonmodified IL-17RC $\left(10 \mu \mathrm{g} \mathrm{mL} \mathrm{m}^{-1}\right)$ was added to determine the binding capacity of truncated IL-17RC peptide. In a separate experiment, plates were coated with nonmodified IL-17RC $\left(10 \mu \mathrm{g} \mathrm{mL}^{-1}\right)$ overnight and then treated with IL-17A or IL-17F $\left(100 \mathrm{ng} \mathrm{mL}^{-1}\right)$ with and without truncated IL-17RC peptide $\left(10 \mu \mathrm{g} \mathrm{mL} \mathrm{m}^{-1}\right)$. The plates were washed and then treated with biotinylated antibodies to IL-17A and IL-17F $\left(0.2 \mu \mathrm{g} \mathrm{mL}^{-1}\right)$ and washed again. The absorbance of each well was measured at $450 \mathrm{~nm}$ by a microplate reader (Thermomax Technologies).

Neutralization of Truncated IL-17RC Peptide: The cells were all placed onto 96-well plates and stimulated with IL-17A or IL-17F $\left(3 \mathrm{ng} \mathrm{mL}^{-1}\right)$ in the presence or absence of truncated IL-17RC peptide $\left(300 \mathrm{ng} \mathrm{mL}^{-1}\right.$ ) for $48 \mathrm{~h}$ or $7 \mathrm{~d}$. First, the levels of IL- 6 and TNF- $\alpha$ were determined in supernatants of MC3T3-E1 or NIH/3T3 at a density of $5 \times 10^{4}$ cells per well after $48 \mathrm{~h}$. In addition, the level of RANKL was further evaluated in supernatants of MC3T3-EI $\left(1 \times 10^{4}\right.$ cells per well) after $7 \mathrm{~d}$. The cytokine concentrations were measured by mouse DuoSet ELISA development system according to the manufacturers' instructions. Then, mouse total MMP-9 ELISA kit was used to determine its secretion from RAW264.7 cells at a density of $5 \times 10^{3}$ cells per well after $7 \mathrm{~d}$.

Coculture System and Osteoclastogenesis Assay: A coculture system for RAW264.7 and MC3T3-E1 was established using Transwell inserts (Corning, USA). ${ }^{[32,33]}$ RAW264.7 cells $\left(3 \times 10^{4}\right.$ cells per well) were seeded in 24-well glass insert plates. MC3T3-E1 cells $\left(1 \times 10^{4}\right.$ cells per well) were 
embedded in the lower compartment of each Transwell. This coculture system was maintained in DMEM with IL-17A or IL-17F $\left(10 \mathrm{ng} \mathrm{mL}^{-1}\right)$ treatment in the presence or absence of truncated IL-17RC $\left(1 \mu \mathrm{g} \mathrm{mL}^{-1}\right)$ for $7 \mathrm{~d}$. The cells were then subjected to TRAP staining using an acid phosphatase kit, and the number of osteoclast-like TRAP-positive cells (multinucleated cells, MNCs $>3$ nuclei) was counted.

Induction of CIA: In the first CIA model, $100 \mu \mathrm{L}$ chick type II collagen dissolved in $0.01 \mathrm{M}$ acetic acid to a concentration of $4 \mathrm{mg} \mathrm{mL}^{-1}$ was emulsified in an equal volume of Freund's complete adjuvant and administered intradermally at the base of the tail of C57BL/ $6 \mathrm{~N}$ mice (15 mice per group). On day 21, a booster emulsion prepared with type II collagen and Freund's incomplete adjuvant was administered intradermally near the primary injection site. ${ }^{[34-36]}$ The truncated IL-17RC peptide $(150 \mu \mathrm{g}, 100 \mu \mathrm{L})$ was injected intraperitoneally three times per week on day 14 after the initial immunization whereas PBS was used as a control. The hind-paw was scored every day and the swelling was measured every $3 \mathrm{~d}$. On day 37 after the first immunization, mice were anesthetized. The clinical scores were assigned as previously described. ${ }^{[36]}$

In the CIA-IL-17A/F mouse model, IL-17A or IL-17F $(200 \mathrm{ng}$ in $10 \mu \mathrm{L}$ PBS) was injected into the knee joints on day 28 and day 32 after the first injection of chick type II collagen. ${ }^{[37-39]}$ The truncated IL-17RC peptide $(150 \mu \mathrm{g}, 100 \mu \mathrm{L})$ was injected intraperitoneally three times per week on day 14 after the initial immunization whereas PBS was used as a control. The knee joints were scored and measured from day 28 . On day 37 after the first immunization, mice were anesthetized. Knee joint damage was scored visually after knee joint skin dissection under intraperitoneal anaesthesia as previously described. ${ }^{[38]}$

Histological Analysis: Murine hind paws and knee joints were removed postmortem, stored in 10\% neutral formalin, decalcified in $20 \%$ ethylenediamine tetraacetic acid (EDTA) for 4 weeks, then dehydrated and embedded in paraffin. Sections were cut along the longitudinal axis, mounted and stained with hematoxylin and eosin (H\&E) or toluidine blue (TB). The severity of inflammatory cell infiltration in joint and cartilage destruction was scored according previously described. ${ }^{[40]}$

Radiographic Analysis: Quantitative analysis of the hind paw and knee joints was performed after sacrifice using a micro-CT system (SKYSCAN 1172; Bruker, Belgium). 3D analysis was performed using CTAn software (Bruker). The fraction of bone surface (BS), bone volume (BV), bone surface/bone volume (BS/BV), percent bone volume (BV/TV), cortex bone mineral density (Ct. BMD), cortex area (Ct. Ar), cortex thickness (Ct. Th), trabecular bone mineral density (Tb. BMD), trabecular number (Tb. N), trabecular thickness (Tb. Th), trabecular separation (Tb. $\mathrm{Sp}$ ), and degree of anisotropy (DA) were analyzed using the built-in software. ${ }^{[41,42]}$

Serological Analysis: Serum cytokines were measured on day 0, 14, 28, and 37 at which times the mice were euthanized. The blood (10-15 drops) was extracted from mouse orbital veins. The concentrations of IL-6, TNF- $\alpha$, MCP-1, IFN- $\gamma$, IL-10, and IL-12p70 in serum were measured using CBA mouse inflammation kits. The concentration of RANKL, OPG or MMP-9 in serum was measured using mouse ELISA kits. All procedures were performed according to the manufacturers' protocols.

Statistical Analysis: The data were analysed using a statistical package (SPSS13.0, Chicago, IL, USA). The Student's t-test or one-way analysis of variance for parametric analysis was utilized for between-group comparisons. A p-value less than 0.05 was considered to be statistically significant.

\section{Supporting Information}

Supporting Information is available from the Wiley Online Library or from the author.

\section{Acknowledgements}

This study was financially supported by the National Nature Science Foundations of China (Grant No. 31370936) and the State Key
Development Program for Basic Research of China (Grant No. 2010CB529106).

Received: June 23, 2016

Revised: August 7, 2016

Published online: October 6, 2016

[1] G. S. Firestein, Nature 2003, 423, 356.

[2] Y. Iwakura, H. Ishigame, S. Saijo, S. Nakae, Immunity 2011, 34, 149.

[3] J. Zupan, M. Jeras, J. Marc, Biochem. Med. 2013, 23, 43.

[4] E. Lubberts, Cytokine 2008, 41, 84.

[5] X. Song, Y. Qian, Cytokine 2013, 62, 175.

[6] M. J. Ruddy, G. C. Wong, X. K. Liu, H. Yamamoto, S. Kasayama, K. L. Kirkwood, S. L. Gaffen, J. Biol. Chem. 2004, 279, 2559.

[7] G. Benedetti, P. Miossec, Eur. J. Immunol. 2014, 44, 339.

[8] B. Schminke, S. Trautmann, B. Mai, N. Miosge, S. Blaschke, Eur. J. Immunol. 2016, 46, 440.

[9] K. Kaneko, R. O. Williams, D. T. Dransfield, A. E. Nixon, A. Sandison, Y. Itoh, Arthritis Rheumatol. 2016, 68, 521.

[10] M. Chabaud, P. Miossec, Arthritis Rheumatol. 2001, 44, 1293.

[11] S. Kitami, H. Tanaka, T. Kawato, N. Tanabe, T. Katono-Tani, F. Zhang, N. Suzuki, Y. Yonehara, M. Maeno, Biochimie 2010, 92, 398.

[12] F. Zhang, H. Tanaka, T. Kawato, S. Kitami, K. Nakai, M. Motohashi, N. Suzuki, C. L. Wang, K. Ochiai, K. Isokawa, M. Maeno, Biochimie 2011, 93, 296.

[13] M. I. Koenders, E. Lubberts, B. Oppers-Walgreen, L. van den Bersselaar, M. M. Helsen, F. E. Di Padova, A. M. Boots, H. Gram, L. A. Joosten, W. B. van den Berg, Am. J. Pathol. 2005, 167, 141.

[14] T. Yago, Y. Nanke, N. Ichikawa, T. Kobashigawa, M. Mogi, N. Kamatani, S. Kotake, J. Cell Biochem. 2009, 108, 947.

[15] A. W. Ho, S. L. Gaffen, Semin. Immunopathol. 2010, 32, 33.

[16] A. Hot, S. Zrioual, M. L. Toh, V. Lenief, P. Miossec, Ann. Rheum. Dis. 2011, 70, 341.

[17] S. L. Gaffen, Nat. Rev. Immunol. 2009, 9, 556.

[18] R. E. Kuestner, D. W. Taft, A. Haran, C. S. Brandt, T. Brender, K. Lum, B. Harder, S. Okada, C. D. Ostrander, J. L. Kreindler, S. J. Aujla, B. Reardon, M. Moore, P. Shea, R. Schreckhise, T. R. Bukowski, S. Presnell, P. Guerra-Lewis, J. Parrish-Novak, J. L. Ellsworth, S. Jaspers, K. E. Lewis, M. Appleby, J. K. Kolls, M. Rixon, J. W. West, Z. Gao, S. D. Levin, J. Immunol. 2007, 179, 5462.

[19] L. G. van Baarsen, M. C. Lebre, D. van der Coelen, S. Aarrass, M. W. Tang, T. H. Ramwadhdoebe, D. M. Gerlag, P. P. Tak, Arthritis Res. Ther. 2014, 16, 426.

[20] T. A. Moseley, D. R. Haudenschild, L. Rose, A. H. Reddi, Cytokine Growth Factor Rev. 2003, 14, 155.

[21] R. Kugyelka, Z. Kohl, K. Olasz, K. Mikecz, T. A. Rauch, T. T. Glant, F. Boldizsar, Mediators Inflammation 2016, 2016, 6145810.

[22] E. Lubberts, M. I. Koenders, B. Oppers-Walgreen, L. van den Bersselaar, R. C. Coenen-de, L. A. Joosten, W. B. van den Berg, Arthritis Rheum. 2004, 50, 650 .

[23] M. Kopf, M. F. Bachmann, B. J. Marsland, Nat. Rev. Drug Discovery 2010, 9, 703.

[24] M. Zaretsky, R. Etzyoni, J. Kaye, L. Sklair-Tavron, A. Aharoni, Chem. Biol. 2013, 20, 202.

[25] C. L. Krieckaert, A. Jamnitski, M. T. Nurmohamed, P. J. Kostense, M. Boers, G. Wolbink, Arthritis Rheum. 2012, 64, 3850.

[26] S. A. Metawi, D. Abbas, M. M. Kamal, M. K. Ibrahim, Clin Rheumatol. 2011, 30, 1201

[27] A. Varas, J. Valencia, F. Lavocat, V. G. Martinez, N. N. Thiam, L. Hidalgo, L. M. Fernandez-Sevilla, R. Sacedon, A. Vicente, P. Miossec, Arthritis Res. Ther. 2015, 17, 192. 
[28] H. B. Hsiao, C. C. Hsieh, J. B. Wu, H. Lin, W. C. Lin, BMC Complementary Altern. Med. 2016, 16, 80.

[29] Y. Iwakura, S. Nakae, S. Saijo, H. Ishigame, Immunol Rev. 2008, 226, 57.

[30] A. Hot, P. Miossec, Ann. Rheum. Dis. 2011, 70, 727.

[31] J. M. Harris, R. B. Chess, Nat. Rev. Drug Discovery 2003, 2, 214.

[32] J. Li, Z. Wan, H. Liu, H. Li, L. Liu, R. Li, Y. Guo, W. Chen, X. Zhang, X. Zhang, Ann. Biomed. Eng. 2013, 41, 2056.

[33] Q. G. Mai, Z. M. Zhang, S. Xu, M. Lu, R. P. Zhou, L. Zhao, C. H. Jia, Z. H. Wen, D. D. Jin, X. C. Bai, J. Cell. Biochem. 2011, 112, 2902.

[34] M. Elhai, G. Chiocchia, C. Marchiol, F. Lager, G. Renault, M. Colonna, G. Bernhardt, Y. Allanore, J. Avouac, J. Inflammation 2015, 12, 9.

[35] J. J. Inglis, E. Simelyte, F. E. McCann, G. Criado, R. O. Williams, Nat. Protoc. 2008, 3, 612.
[36] M. I. Ramos, O. N. Karpus, P. Broekstra, S. Aarrass, S. E. Jacobsen, P. P. Tak, M. C. Lebre, Ann. Rheum. Dis. 2015, 74, 211

[37] J. H. Lee, M. L. Cho, J. I. Kim, Y. M. Moon, H. J. Oh, G. T. Kim, S. Ryu, S. H. Baek, S. H. Lee, H. Y. Kim, S. I. Kim, J. Rheumatol. 2009, 36, 684

[38] S. H. Baek, S. G. Lee, Y. E. Park, G. T. Kim, C. D. Kim, S. Y. Park, Inflammation Res. 2012, 61, 1339.

[39] Y. E. Park, Y. J. Woo, S. H. Park, Y. M. Moon, H. J. Oh, J. I. Kim, H. S. Jin, S. H. Baek, G. T. Kim, J. H. Lee, M. L. Cho, S. I. Kim, Immunol. Lett. 2011, 140, 97.

[40] H. Yuan, H. Qian, S. Liu, X. Zhang, S. Li, W. Wang, Z. Li, J. Jia W. Zhao, Biomaterials 2012, 33, 8177.

[41] K. Al-Hezaimi, S. Ramalingam, M. Al-Askar, A. S. ArRejaie, N. Nooh, F. Jawad, A. Aldahmash, M. Atteya, C. Y. Wang, Int. J. Oral Sci. 2016, 8, 7.

[42] Y. H. Kim, J. S. Kang, J. Vet. Sci. 2015, 16, 165. 